\title{
An extended Halanay inequality with unbounded coefficient functions on time scales
}

Boqun Ou', Quanwen Lin², Feifei Du ${ }^{3}$ and Baoguo $\mathrm{Jia}^{3}$

\section{"Correspondence:}

linquanwen@126.com

${ }^{2}$ Department of Mathematics,

Guangdong University of

Petrochemical Technology,

Maoming, 525000, China

Full list of author information is

available at the end of the article

\begin{abstract}
In this paper, we obtain an extended Halanay inequality with unbounded coefficient functions on time scales, which extends an earlier result in Wen et al. (J. Math. Anal. Appl. 347:169-178, 2008). Two illustrative examples are also given.
\end{abstract}

Keywords: time scales; delay dynamic equation; coefficient functions; inequality

\section{Introduction and preliminaries}

As is well known, Halanay-type differential inequalities have been very useful in the stability analysis of time-delay systems and these have led to some interesting new stability conditions (see [1-4] and the references therein).

In [3], Halanay proved the following inequality.

Lemma 1.1 (Halanay's inequality) If

$$
x^{\prime}(t) \leq-\alpha x(t)+\beta \sup _{s \in[t-\tau, t]} x(s), \quad \text { for } t \geq t_{0}, \tau>0
$$

and $\alpha>\beta>0$, then there exist $\gamma>0$ and $K>0$ such that

$$
x(t) \leq K e^{-\gamma\left(t-t_{0}\right)}, \quad \text { for } t \geq t_{0} .
$$

In [5], Baker and Tang obtained the following Halanay-type inequality with unbounded coefficient functions.

Lemma 1.2 (see [5]) Let $x(t)>0, t \in(-\infty,+\infty)$, and

$$
\begin{aligned}
& \frac{d x(t)}{d t} \leq-a(t) x(t)+b(t) \sup _{t-\tau(t) \leq s \leq t} x(s), \quad t>t_{0}, \\
& x(t)=|\varphi(t)|, \quad t \leq t_{0},
\end{aligned}
$$

(c) The Author(s) 2016. This article is distributed under the terms of the Creative Commons Attribution 4.0 International License (http://creativecommons.org/licenses/by/4.0/), which permits unrestricted use, distribution, and reproduction in any medium, provided you give appropriate credit to the original author(s) and the source, provide a link to the Creative Commons license, and indicate if changes were made. 
where $\varphi(t)$ is bounded and continuous for $t \leq t_{0}$, and $a(t) \geq 0, b(t) \geq 0$ for $t \in\left[t_{0}, \infty\right), \tau(t) \geq$ 0 and $t-\tau(t) \rightarrow \infty$ as $t \rightarrow \infty$. If there exists $\sigma>0$ such that

$$
-a(t)+b(t) \leq-\sigma<0 \quad \text { for } t \geq t_{0}
$$

then

(i) $\quad x(t) \leq\|\varphi\|^{\left(-\infty, t_{0}\right]}, \quad t \geq t_{0}$ and (ii) $x(t) \rightarrow 0 \quad$ as $t \rightarrow \infty$,

where $\|\varphi\|^{\left(-\infty, t_{0}\right]}=\sup _{t \in\left(-\infty, t_{0}\right]}|\varphi(t)|<\infty$.

In [1], Wen et al. obtained an extension of Lemma 1.2.

In this paper, we extend the main results of [5] to time scale. As an application, we consider the stability of the following delay dynamic equation:

$$
\left\{\begin{array}{l}
x^{\Delta}(t)=-a(t) x^{\sigma}(t)+b(t) x(t-\tau(t))+c(t), \quad t \in\left[t_{0},+\infty\right)_{\mathbb{T}}, \\
x(s)=|\varphi(s)| \text { for } s \in\left(-\infty, t_{0}\right]_{\mathbb{T}}
\end{array}\right.
$$

where $\varphi(s)$ is bounded rd-continuous for $s \in\left(-\infty, t_{0}\right]_{\mathbb{T}}$ and $\tau(t), a(t), b(t), c(t)$ are nonnegative, rd-continuous functions for $t \in\left[t_{0}, \infty\right)_{\mathbb{T}}$ and $c(t)$ is bounded. We prove that the zero solution of the delay difference equation

$$
\triangle x(n)=-2(n+1) x(n+1)+\frac{n^{2}}{2 n+1} x(n-2), \quad n \geq 0,
$$

is stable.

For completeness, we introduce the following concepts related to the notions of time scales. We refer to [6] for additional details concerning the calculus on time scales.

Definition 1.1 (see [6]) A function $h: \mathbb{T} \rightarrow \mathbb{R}$ is said to be regressive provided $1+$ $\mu(t) h(t) \neq 0$ for all $t \in \mathbb{T}^{\kappa}$, where $\mu(t)=\sigma(t)-t$. The set of all regressive rd-continuous functions $\varphi: \mathbb{T} \rightarrow \mathbb{R}$ is denoted by $\mathfrak{R}$ while the set $\mathfrak{R}^{+}$is given by $\mathfrak{R}^{+}=\{\varphi \in \mathfrak{R}: 1+$ $\mu(t) \varphi(t)>0$ for all $t \in \mathbb{T}\}$. If $\varphi \in \mathfrak{R}$, the exponential function is defined by

$$
e_{\varphi}(t, s)=\exp \left(\int_{s}^{t} \xi_{\mu(r)}(\varphi(r)) \Delta r\right), \quad \text { for } t \in \mathbb{T}, s \in \mathbb{T}^{\kappa},
$$

where $\xi_{\mu(s)}$ is the cylinder transformation given by

$$
\xi_{\mu(r)}(\varphi(r)):= \begin{cases}\frac{1}{\mu(r)} \log (1+\mu(r) \varphi(r)), & \mu(r)>0, \\ \varphi(r), & \mu(r)=0,\end{cases}
$$

and some properties of the exponential function are given in the following lemma.

Lemma 1.3 (see [7]) Let $\varphi \in \Re$, Then

(i) $e_{0}(s, t) \equiv 1, e_{\varphi}(t, t) \equiv 1$ and $e_{\varphi}(\sigma(t), s)=(1+\mu(t) \varphi(t)) e_{\varphi}(t, s)$;

(ii) $\frac{1}{e_{\varphi}(t, s)}=e_{\ominus \varphi}(t, s)$, where $\ominus \varphi(t)=-\frac{\varphi(t)}{1+\mu(t) \varphi(t)}$;

(iii) $\left(\frac{1}{e_{\varphi}(t, s)}\right)^{\Delta}=-\frac{\varphi(t)}{e_{\varphi}(\sigma(t), s)}$; 
(iv) $\left[e_{\varphi}(c, t)\right]^{\Delta}=-\varphi(t) e_{\varphi}(c, \sigma(t))$, where $c \in \mathbb{T}$;

(v) $e_{p}(t, s)=\frac{1}{e_{p}(s, t)}=e_{\ominus p}(s, t)$.

Lemma 1.4 (see [8]) For a nonnegative $\varphi$ with $-\varphi \in \mathfrak{R}^{+}$, we have the inequalities

$$
1-\int_{s}^{t} \varphi(u) \Delta u \leq e_{-\varphi}(t, s) \leq \exp \left\{-\int_{s}^{t} \varphi(u) \Delta u\right\} \text { for all } t \geq s .
$$

If $\varphi$ is rd-continuous and nonnegative, then

$$
1+\int_{s}^{t} \varphi(u) \Delta u \leq e_{\varphi}(t, s) \leq \exp \left\{\int_{s}^{t} \varphi(u) \Delta u\right\} \text { for all } t \geq s .
$$

Remark 1.1 If $\varphi \in \mathfrak{R}^{+}$and $\varphi(r)>0$ for all $r \in[s, t]_{\mathbb{T}}$, then

$$
e_{\varphi}(t, r) \leq e_{\varphi}(t, s) \quad \text { and } \quad e_{\varphi}(a, b)<1 \quad \text { for } s \leq a<b \leq t .
$$

Proof By $\varphi(r)>0, \varphi \in \mathfrak{R}^{+}$and Lemma 1.3(iv) we have $\left[e_{\varphi}(c, t)\right]^{\Delta}=-\varphi(t) e_{\varphi}(c, \sigma(t))<0$, so

$$
e_{\varphi}(t, r) \leq e_{\varphi}(t, s)
$$

Since $a<b$, from the above result, we have

$$
e_{\varphi}(a, b)<e_{\varphi}(a, a)=1
$$

\section{Main results}

Throughout this paper, we assume that the following conditions hold:

$\left(\mathrm{H}_{1}\right)$ Let $x(t)$ be a nonnegative right-dense function satisfying

$$
\left\{\begin{aligned}
& x^{\Delta}(t) \leq-a(t) x(t)+b(t) \sup _{t-\tau(t) \leq s \leq t} x(s)+c(t) \\
&+d(t) \int_{0}^{\infty} K(t, s) x(t-s) \Delta s, \quad t \in\left[t_{0}, \infty\right), \\
& x(t)=|\varphi(t)|, \quad t \in\left(-\infty, t_{0}\right]
\end{aligned}\right.
$$

where $\varphi(t)$ is bounded rd-continuous for $t \in\left(-\infty, t_{0}\right]_{\mathbb{T}}$ and $\sup _{t \leq t_{0}}|\varphi(t)|=M$.

$\left(\mathrm{H}_{2}\right) a(t), b(t), c(t), \tau(t)$ are nonnegative, rd-continuous functions for $t \in\left[t_{0}, \infty\right)_{\mathbb{T}}$ and $c(t)$ is bounded, such that $\sup _{t \geq t_{0}} c(t)=\bar{c}, \lim _{t \rightarrow \infty}(t-\tau(t))=+\infty$.

$\left(\mathrm{H}_{3}\right)$ There exists $\delta>0$ such that $a(t)-b(t)-d(t) \int_{0}^{\infty} K(t, s) \Delta s>\delta>0$, for $t \in\left[t_{0}, \infty\right)_{\mathbb{T}}$, where the delay kernel $K(t, s)$ is a nonnegative, rd-continuous for $(t, s) \in \mathbb{T} \times[0, \infty)$ and satisfies $\forall t \in \mathbb{T}, \int_{0}^{\infty} K(t, s) \triangle s<\infty$.

Theorem 2.1 Assume that $\left(\mathrm{H}_{1}\right)-\left(\mathrm{H}_{3}\right)$ and $-a(t) \in \mathfrak{R}^{+}$hold, then we have

(i)

$$
x(t) \leq \frac{\bar{c}}{\delta}+M, \quad t \in\left[t_{0},+\infty\right) .
$$

If we assume further that $d(t)=0$ in $\left(\mathrm{H}_{1}\right),\left(\mathrm{H}_{3}\right)$ and there exists $0<\kappa<1$ such that

$$
\kappa a(t)-b(t)>0 \quad \text { for } t \in\left[t_{0},+\infty\right)_{\mathbb{T}},
$$

then we have 
(ii) for any given $\epsilon>0$, there exists $\tilde{t}=\widetilde{t}(M, \epsilon)>t_{0}$, such that

$$
x(t) \leq \frac{\bar{c}}{\delta}+\epsilon, \quad t \in[\tilde{t}, \infty)
$$

Proof We now consider the following two cases successively.

Case 1. $\bar{c}>0$.

Proof of Theorem 2.1(i).

For any $\varepsilon>1$, we have from $\left(\mathrm{H}_{1}\right)$

$$
\forall t \leq t_{0}, \quad x(t)=|\varphi(t)| \leq \sup _{t \leq t_{0}}|\varphi(t)|=M<\frac{\bar{c}}{\delta}+\varepsilon M,
$$

from this we shall deduce that

$$
\forall t \geq t_{0}, \quad x(t)<\frac{\bar{c}}{\delta}+\varepsilon M
$$

To prove (2.5), let $t_{1}=\sup \left\{t \mid x(s) \leq \frac{\bar{c}}{\delta}+\varepsilon M, s \in\left[t_{0}, t\right]_{\mathbb{T}}\right\}>t_{0}$, we will show $t_{1}=\infty$.

Suppose $t_{1}<\infty$. Clearly we have $x\left(t_{1}\right) \leq \frac{\bar{c}}{\delta}+\varepsilon M$.

In fact, suppose that $x\left(t_{1}\right) \leq \frac{\bar{c}}{\delta}+\varepsilon M$ fails, then we have $x\left(t_{1}\right)>\frac{\bar{c}}{\delta}+\varepsilon M$.

If $t_{1}$ is left-dense, there is $\left\{t_{n}\right\}$ satisfying: $t_{n}<t_{1}, t_{n} \rightarrow t_{1}(n \rightarrow \infty)$, and $x\left(t_{n}\right) \leq \frac{\bar{c}}{\delta}+\varepsilon M$, we have $x\left(t_{1}\right)=\lim _{n \rightarrow \infty} x\left(t_{n}\right) \leq \frac{\bar{c}}{\delta}+\varepsilon M$, which contradicts $x\left(t_{1}\right)>\frac{\bar{c}}{\delta}+\varepsilon M$.

If $t_{1}$ is left-scattered, $\rho\left(t_{1}\right)<t_{1}$ and $x\left(\rho\left(t_{1}\right)\right) \leq \frac{\bar{c}}{\delta}+\varepsilon M ; x\left(t_{1}\right)>\frac{\bar{c}}{\delta}+\varepsilon M$, then we have sup $\left\{t \mid x(s)<\frac{\bar{c}}{\delta}+\varepsilon M, s \in\left[t_{0}, t\right]\right\}=\rho\left(t_{1}\right)<t_{1}$, which contradicts the definition of $t_{1}$.

Therefore we can suppose $t_{1}<\infty, x\left(t_{1}\right) \leq \frac{\bar{c}}{\delta}+\varepsilon M$. We will discuss two cases:

Case 1.1. Suppose $x\left(t_{1}\right)=\frac{\bar{c}}{\delta}+\varepsilon M, t_{1}>t_{0}$,

$$
\forall t \in\left[t_{0}, t_{1}\right)_{\mathbb{T}}, \quad x(t) \leq \frac{\bar{c}}{\delta}+\varepsilon M, \quad x\left(t_{1}\right)=\frac{\bar{c}}{\delta}+\varepsilon M .
$$

Clearly we have $x^{\Delta}\left(t_{1}\right) \geq 0$. In fact, suppose that $x^{\Delta}\left(t_{1}\right) \geq 0$ fails, then we have $x^{\Delta}\left(t_{1}\right)<0$.

If $t_{1}$ is right-dense, $\forall s>t_{1}$, from $x^{\Delta}\left(t_{1}\right)=\lim _{s \rightarrow t_{1}^{+}} \frac{x\left(t_{1}\right)-x(s)}{t_{1}-s}<0$, we get $x(s)<x\left(t_{1}\right)=\frac{\bar{c}}{\delta}+\varepsilon M$, which contradicts the definition of $t_{1}$.

If $t_{1}$ is right-scattered, from $x^{\Delta}\left(t_{1}\right)=\frac{x\left(\sigma\left(t_{1}\right)\right)-x\left(t_{1}\right)}{\mu\left(t_{1}\right)}<0$, we get $x\left(\sigma\left(t_{1}\right)\right)<x\left(t_{1}\right)=\frac{\bar{c}}{\delta}+\varepsilon M$, which contradicts the definition of $t_{1}$.

We have from (2.6), $\left(\mathrm{H}_{1}\right)$, and $\left(\mathrm{H}_{3}\right)$

$$
\begin{aligned}
x^{\Delta}\left(t_{1}\right) & \stackrel{\left(\mathrm{H}_{1}\right)}{\leq}-a\left(t_{1}\right) x\left(t_{1}\right)+b\left(t_{1}\right) \sup _{t_{1}-\tau\left(t_{1}\right) \leq s \leq t_{1}} x(s)+c\left(t_{1}\right)+d\left(t_{1}\right) \int_{0}^{\infty} K\left(t_{1}, s\right) x\left(t_{1}-s\right) \triangle s \\
& \stackrel{(2.6)}{=}-a\left(t_{1}\right)\left(\frac{\bar{c}}{\delta}+\varepsilon M\right)+b\left(t_{1}\right) \sup _{t_{1}-\tau\left(t_{1}\right) \leq s \leq t_{1}} x(s)+c\left(t_{1}\right)+d\left(t_{1}\right) \int_{0}^{\infty} K\left(t_{1}, s\right) x\left(t_{1}-s\right) \triangle s \\
& \stackrel{(2.6)}{\leq}-\left(a\left(t_{1}\right)-b\left(t_{1}\right)+d\left(t_{1}\right) \int_{0}^{\infty} K\left(t_{1}, s\right) \triangle s\right)\left(\frac{\bar{c}}{\delta}+\varepsilon M\right)+\bar{c} \\
& \stackrel{\left(\mathrm{H}_{3}\right)}{<}-\delta\left(\frac{\bar{c}}{\delta}+\varepsilon M\right)+\bar{c}=-\delta \varepsilon M<0
\end{aligned}
$$

which contradicts $x^{\triangle}\left(t_{1}\right) \geq 0$. 
Case 1.2. Suppose $x\left(t_{1}\right)<\frac{\bar{c}}{\delta}+\varepsilon M$. In this case, $t_{1}$ must be right-scattered, for otherwise if $t_{1}$ is right-dense, there exists $\epsilon_{1}$ sufficiently small so that $x(t)<\frac{\bar{c}}{\delta}+\varepsilon M$, for $t \in\left[t_{1}, t_{1}+\epsilon_{1}\right]_{\mathbb{T}}$. Therefore, $x(t) \leq \frac{\bar{c}}{\delta}+\varepsilon M$, for $t \in\left[t_{0}, t_{1}+\epsilon_{1}\right]_{\mathbb{T}}$. This contradicts the definition of $t_{1}$. Hence, since $t_{1}$ is right-scattered, we have

$$
x\left(\sigma\left(t_{1}\right)\right)>\frac{\bar{c}}{\delta}+\varepsilon M \quad \text { and } \quad x(t) \leq \frac{\bar{c}}{\delta}+\varepsilon M \quad \text { for all } t \leq t_{1}<\sigma\left(t_{1}\right)
$$

We have from $(2.8)$ and $\left(\mathrm{H}_{1}\right)$

$$
\begin{aligned}
& \frac{x\left(\sigma\left(t_{1}\right)\right)-x\left(t_{1}\right)}{\mu\left(t_{1}\right)}=x^{\Delta}\left(t_{1}\right) \\
& \stackrel{\left(\mathrm{H}_{1}\right)}{\leq}-a\left(t_{1}\right) x\left(t_{1}\right)+b\left(t_{1}\right) \sup _{t_{1}-\tau\left(t_{1}\right) \leq s \leq t_{1}} x(s)+c\left(t_{1}\right) \\
&+d\left(t_{1}\right) \int_{0}^{\infty} K\left(t_{1}, s\right) x\left(t_{1}-s\right) \triangle s \\
& \stackrel{(2.8)}{<}-a\left(t_{1}\right) x\left(t_{1}\right)+\left(b\left(t_{1}\right)+d\left(t_{1}\right) \int_{0}^{\infty} K\left(t_{1}, s\right) \triangle s\right)\left(\frac{\bar{c}}{\delta}+\varepsilon M\right)+\bar{c} .
\end{aligned}
$$

By (2.8), (2.9), $\left(\mathrm{H}_{3}\right)$, and $1-\mu(t) a(t)>0, t \in \mathbb{T}$, we get

$$
\begin{aligned}
\frac{\bar{c}}{\delta}+\varepsilon M< & x\left(\sigma\left(t_{1}\right)\right) \\
\stackrel{(2.9)}{<}\left(1-\mu\left(t_{1}\right) a\left(t_{1}\right)\right) x\left(t_{1}\right)+\mu\left(t_{1}\right)\left(b\left(t_{1}\right)+d\left(t_{1}\right) \int_{0}^{\infty} K\left(t_{1}, s\right) \triangle s\right)\left(\frac{\bar{c}}{\delta}+\varepsilon M\right) & \\
& +\mu\left(t_{1}\right) \bar{c} \\
\stackrel{(2.8)}{<} & \left(1-\mu\left(t_{1}\right) a\left(t_{1}\right)+\mu\left(t_{1}\right) b\left(t_{1}\right)+\mu\left(t_{1}\right) d\left(t_{1}\right) \int_{0}^{\infty} K\left(t_{1}, s\right) \triangle s\right)\left(\frac{\bar{c}}{\delta}+\varepsilon M\right) \\
& +\mu\left(t_{1}\right) \bar{c} \\
\stackrel{\left(\mathrm{H}_{3}\right)}{\leq} & \left(1-\delta \mu\left(t_{1}\right)\right)\left(\frac{\bar{c}}{\delta}+\varepsilon M\right)+\mu\left(t_{1}\right) \bar{c}=\frac{\bar{c}}{\delta}+\varepsilon M-\delta \varepsilon M \mu\left(t_{1}\right),
\end{aligned}
$$

which leads to a contradiction.

Hence the inequality (2.5) must hold.

Since $\varepsilon>1$ is arbitrary, we let $\varepsilon \rightarrow 1^{+}$and obtain

$$
\forall t \geq t_{0}, \quad x(t) \leq \frac{\bar{c}}{\delta}+M
$$

Proof of Theorem 2.1(ii).

If $M=0$, it is evident from (2.1) that (2.3) holds. Now we assume $M>0$. Let $\lim \sup _{t \rightarrow \infty} x(t)=\alpha$, then $0 \leq \alpha \leq \frac{\bar{c}}{\delta}+M$. Now we prove that $\alpha \leq \frac{\bar{c}}{\delta}$.

Suppose this is not true, i.e. $\alpha>\frac{\bar{c}}{\delta}$, then we can choose $\varepsilon_{2}>0$ such that $\alpha=\frac{\bar{c}}{\delta}+\varepsilon_{2}$.

Since $\tau(t) \geq 0$, and $\lim _{t \rightarrow \infty}(t-\tau(t))=+\infty$, we have $\limsup _{t \rightarrow \infty} \sup _{t-\tau(t) \leq s \leq t} x(s)=\alpha$.

Clearly, there exists a sufficiently large $T>0$ and $T$ is fixed, such that

$$
\lambda:=\kappa+(1-\kappa) \exp (-\delta T)<1
$$


Taking $\theta: 0<\theta<\frac{1-\lambda}{1+\lambda} \varepsilon_{2}$, using the properties of the superior limits we see that there exists a sufficiently large $t^{*}>t_{0}$, such that

$$
\left\{\begin{array}{l}
x\left(t^{*}\right)>\alpha-\theta, \\
x(t)<\alpha+\theta, \quad t \in\left[t^{*}-T, t^{*}\right] \\
\sup _{t-\tau(t) \leq s \leq t} x(s) \leq \alpha+\theta, \quad t \in\left[t^{*}-T, t^{*}\right] .
\end{array}\right.
$$

On the other hand, it follows from $\left(\mathrm{H}_{1}\right)$ and $\left(\mathrm{H}_{3}\right)$ that

$$
\begin{aligned}
x^{\Delta}(t) & \stackrel{\left(\mathrm{H}_{1}\right)}{\leq}-a(t) x(t)+b(t) \sup _{t-\tau(t) \leq s \leq t} x(s)+c(t) \\
& \stackrel{\left(\mathrm{H}_{3}\right)}{\leq}-a(t) x(t)+b(t) \sup _{t-\tau(t) \leq s \leq t} x(s)+\frac{a(t)-b(t)}{\delta} \bar{c} \\
& =-a(t)\left(x(t)-\frac{\bar{c}}{\delta}\right)+b(t) \sup _{t-\tau(t) \leq s \leq t}\left(x(s)-\frac{\bar{c}}{\delta}\right) .
\end{aligned}
$$

Denote $y(t)=x(t)-\frac{\bar{c}}{\delta}$, and (2.13) implies that

$$
\left\{\begin{array}{l}
y\left(t^{*}\right)=x\left(t^{*}\right)-\frac{\bar{c}}{\delta}>\alpha-\theta-\frac{\bar{c}}{\delta}=\varepsilon_{2}-\theta, \\
y(t)=x(t)-\frac{\bar{c}}{\delta} \leq \alpha+\theta-\frac{\bar{c}}{\delta}=\varepsilon_{2}+\theta, \quad t \in\left[t^{*}-T, t^{*}\right] \\
\sup _{t-\tau(t) \leq s \leq t} y(s)=\sup _{t-\tau(t) \leq s \leq t}\left(x(s)-\frac{\bar{c}}{\delta}\right) \leq \alpha+\theta-\frac{\bar{c}}{\delta}=\varepsilon_{2}+\theta \\
\quad t \in\left[t^{*}-T, t^{*}\right] .
\end{array}\right.
$$

By (2.2), (2.14), (2.15), and $y(t)=x(t)-\frac{\bar{c}}{\delta}$, we have

$$
\begin{gathered}
y^{\triangle}(t) \stackrel{(2.14)}{\leq}-a(t) y(t)+b(t) \sup _{t-\tau(t) \leq s \leq t} y(s) \\
\stackrel{(2.15)}{\leq}-a(t) y(t)+\left(\varepsilon_{2}+\theta\right) b(t) \\
\stackrel{(2.2)}{<}-a(t) y(t)+\kappa\left(\varepsilon_{2}+\theta\right) a(t),
\end{gathered}
$$

which implies

$$
\left(y(t)-\kappa\left(\varepsilon_{2}+\theta\right)\right)^{\Delta} \leq-a(t)\left(y(t)-\kappa\left(\varepsilon_{2}+\theta\right)\right)
$$

then we have

$$
\left(\frac{y(t)-\kappa\left(\varepsilon_{2}+\theta\right)}{e_{-a}\left(t, t_{0}\right)}\right)^{\Delta}=\frac{\left(y(t)-\kappa\left(\varepsilon_{2}+\theta\right)\right)^{\Delta}+a(t)\left(y(t)-\kappa\left(\varepsilon_{2}+\theta\right)\right)}{e_{-a}\left(\sigma(t), t_{0}\right)} \stackrel{(2.17)}{\leq} 0,
$$

where we used the property of the exponential function: if $p \in \mathfrak{R}^{+}$and $t_{0} \in \mathbb{T}$, then $e_{p}\left(t, t_{0}\right)>0$ for all $t \in \mathbb{T}$.

Integrating both sides of (2.18) from $t^{*}-T$ to $t^{*}$ and by (1.11) we obtain

$$
\begin{aligned}
(1-\kappa)\left(\varepsilon_{2}+\theta\right)-2 \theta & =\varepsilon_{2}-\theta-\kappa\left(\varepsilon_{2}+\theta\right) \\
& <y\left(t^{*}\right)-\kappa\left(\varepsilon_{2}+\theta\right)
\end{aligned}
$$




$$
\begin{aligned}
& \stackrel{\text { (Lemma } 1.3(\mathrm{v}))}{\leq} e_{-a}\left(t^{*}, t^{*}-T\right)\left[y\left(t^{*}-T\right)-\kappa\left(\varepsilon_{2}+\theta\right)\right] \\
& \stackrel{(1.11),(2.15)}{\leq}\left(\varepsilon_{2}+\theta-\kappa\left(\varepsilon_{2}+\theta\right)\right) \exp \left(-\int_{t^{*}-T}^{t^{*}} a(u) \Delta u\right) \\
& <(1-\kappa)\left(\varepsilon_{2}+\theta\right) \exp (-\delta T)
\end{aligned}
$$

where we used $a(t) \geq a(t)-b(t) \geq \delta>0$ in the last step.

By (2.19), we have

$$
\theta \geq \frac{1-[\kappa+(1-\kappa) \exp (-\delta T)]}{1+[\kappa+(1-\kappa) \exp (-\delta T)]} \varepsilon_{2}=\frac{1-\lambda}{1+\lambda} \varepsilon_{2}
$$

This contradicts the choice of $\theta$, so we get $\alpha \leq \frac{\bar{c}}{\delta}$. From the definition of the superior limits we obtain (2.3).

Case 2. $\bar{c}=0$.

If only we replace $\bar{c}$ in the proof of Case 1 by $\bar{c}+\epsilon_{3}$ for any given $\epsilon_{3}>0$, then let $\epsilon_{3} \rightarrow 0^{+}$, we find that (2.1) and (2.3) hold.

A combination of Cases 1 and 2 completes the proof of Theorem 2.1.

Remark 2.1 When $M=0$, from (2.7), $\left(\mathrm{H}_{3}\right)$ must have the form that there exists $\delta>0$ such that

$$
a(t)-b(t)-d(t) \int_{0}^{\infty} K(t, s) \triangle s>\delta>0, \quad \text { for } t \in\left[t_{0}, \infty\right)_{\mathbb{T}}
$$

When $M>0,\left(\mathrm{H}_{3}\right)$ may have the form that there exists $\delta>0$ such that

$$
a(t)-b(t)-d(t) \int_{0}^{\infty} K(t, s) \triangle s \geq \delta>0, \quad \text { for } t \in\left[t_{0}, \infty\right)_{\mathbb{T}} .
$$

Similarly, in [1] when $G=0,(2.10)$ must have the form that

$$
\alpha(t)+\beta(t)<-\sigma<0 \quad \text { for } t \geq t_{0}
$$

when $G>0,(2.10)$ may have the form that

$$
\alpha(t)+\beta(t) \leq-\sigma<0 \quad \text { for } t \geq t_{0} .
$$

Theorem 2.1 can be regarded as the extension of the main theorem of [5], Theorem 2.3 of [1].

\section{Applications and examples}

Consider the delay dynamic equation

$$
\left\{\begin{array}{l}
x^{\triangle}(t)=-a(t) x^{\sigma}(t)+b(t) x(t-\tau(t))+c(t)+d(t) \int_{0}^{\infty} K(t, s) x(t-s) \triangle s, \\
\quad t \in\left[t_{0},+\infty\right)_{\mathbb{T}} \\
x(t)=|\varphi(t)| \text { for } t \in\left(-\infty, t_{0}\right]_{\mathbb{T}}
\end{array}\right.
$$


where $\varphi(t)$ is bounded rd-continuous for $s \in\left(-\infty, t_{0}\right]_{\mathbb{T}}$ and $\tau(t), a(t), b(t), c(t), d(t)$ are nonnegative, rd-continuous functions for $t \in\left[t_{0}, \infty\right)_{\mathbb{T}}$ and $c(t)$ is bounded,

$$
\sup _{t \leq t_{0}}|\varphi(t)|=M, \quad \sup _{t \geq t_{0}} c(t)=\bar{c}, \quad \lim _{t \rightarrow \infty}(t-\tau(t))=+\infty .
$$

Assume there exists $\delta>0$ such that

$$
a(t)-b(t)-d(t) \int_{0}^{\infty} K(t, s) \triangle s>\delta>0 \quad \text { for } t \in\left[t_{0}, \infty\right)_{\mathbb{T}},
$$

where the delay kernel $K(t, s)$ is a nonnegative, rd-continuous for $(t, s) \in[0, \infty)_{\mathbb{T}} \times[0, \infty)_{\mathbb{T}}$.

From (3.1), we have

$$
\begin{aligned}
x(t)= & x\left(t_{0}\right) e_{\ominus a}\left(t, t_{0}\right) \\
& +\int_{t_{0}}^{t} e_{\ominus a}(t, s)\left[b(s) x(s-\tau(s))+c(s)+d(s) \int_{0}^{\infty} K(s, v) x(s-v) \Delta v\right] \Delta s .
\end{aligned}
$$

Let the functions $y(t)$ be defined as follows: $y(t)=|x(t)|$ for $t \in\left(-\infty, t_{0}\right]_{\mathbb{T}}$, and

$$
\begin{aligned}
y(t)= & \left|x\left(t_{0}\right)\right| e_{\ominus a}\left(t, t_{0}\right) \\
& +\int_{t_{0}}^{t} e_{\ominus a}(t, s)\left[b(s)|x(s-\tau(s))|+c(s)+d(s) \int_{0}^{\infty} K(s, v)|x(s-v)| \Delta v\right] \triangle s,
\end{aligned}
$$

for $t>t_{0}$. Then we have $|x(t)| \leq y(t)$, for all $t \in(-\infty,+\infty)_{\mathbb{T}}$.

By [9], Theorem 5.37, we get

$$
\begin{aligned}
y^{\Delta}(t)= & \ominus a(t)\left\{\left|x\left(t_{0}\right)\right| e_{\ominus a}\left(t, t_{0}\right)+\int_{t_{0}}^{t} e_{\ominus a}(t, s)[b(s)|x(s-\tau(s))|+c(s)\right. \\
& \left.\left.+d(s) \int_{0}^{\infty} K(s, v)|x(s-v)| \Delta v\right] \triangle s\right\} \\
& +e_{\ominus a}(\sigma(t), t)\left\{b(t)|x(t-\tau(t))|+c(t)+d(t) \int_{0}^{\infty} K(t, v)|x(t-v)| \Delta v\right\} \\
\leq & \frac{1}{1+\mu(t) a(t)}\left\{-a(t) y(t)+b(t) \sup _{t-\tau(t) \leq \theta \leq t} y(\theta)+c(t)\right. \\
& \left.+d(t) \int_{0}^{\infty} K(t, v) y(t-v) \triangle v\right\}, \quad t \in\left[t_{0}, \infty\right)_{\mathbb{T}} .
\end{aligned}
$$

Example 1 Let $\mathbb{T}=\mathbb{R}^{+}$, then system $\left(\mathrm{H}_{1}\right)$ is expressed as

$$
\left\{\begin{aligned}
x^{\prime}(t) \leq & -a(t) x(t)+b(t) \sup _{t-\tau(t) \leq s \leq t} x(s)+c(t) \\
& +d(t) \int_{0}^{\infty} K(t, s) x(t-s) d s, \quad t \geq 0 \\
x(t)= & |\varphi(t)|, \quad t \leq 0
\end{aligned}\right.
$$

where $\varphi(t)$ is bounded continuous for $t \in(-\infty, 0]$ and $\sup _{t \leq 0}|\varphi(t)|=M$. 
We choose some explicit nonnegative, continuous functions for $a(t), b(t), c(t), d(t), \tau(t)$, $K(t, s)$. Let

$$
\begin{aligned}
& a(t)=\frac{(t+1)^{2}}{t+2}, \quad b(t)=\frac{t^{2}+t}{2 e(t+2)}, \quad c(t)=\left(\frac{t+2}{t+1}\right)^{t}, \quad d(t)=\frac{t}{e\left(2-e^{-t^{2}}\right) \sqrt{\pi}}, \\
& K(t, s)=(2-\cos 2 t s) e^{-s^{2}}, \quad(t, s) \in[0, \infty) \times[0, \infty), \tau(t)<t \text { and } \lim _{t \rightarrow \infty}(t-\tau(t))=+\infty .
\end{aligned}
$$

Obviously, $a(t), b(t), d(t)$ are unbounded for $t \geq 0$ and $\sup _{t \geq 0} c(t)=\bar{c}=e$.

(1) $\forall t \in[0, \infty), g(t):=\int_{0}^{\infty} K(t, s) d s=\int_{0}^{\infty}(2-\cos 2 t s) e^{-s^{2}} d s$, then since $\forall(t, s) \in[0, \infty) \times$ $[0, \infty)$

$$
\begin{aligned}
& |K(t, s)| \leq 3 e^{-s^{2}} \text { and }\left|\frac{\partial K(t, s)}{\partial t}\right|=\left|2 s e^{-s^{2}} \sin 2 t s\right| \leq 2 s e^{-s^{2}} \\
& \int_{0}^{\infty} e^{-s^{2}} d s=\frac{\sqrt{\pi}}{2}=g(0) \text { and } \int_{0}^{\infty} 2 s e^{-s^{2}} d s=1
\end{aligned}
$$

we have $g(t)=\int_{0}^{\infty} K(t, s) d s$ is convergent for $t \in[0, \infty)$ and $\int_{0}^{\infty} K_{t}(t, s) d s$ is uniformly convergent for $t \in[0, \infty)$.

So

$$
g^{\prime}(t)=\int_{0}^{\infty} K_{t}(t, s) d s=\int_{0}^{\infty} 2 s e^{-s^{2}} \sin 2 t s d s=-2 \operatorname{tg}(t)+2 \sqrt{\pi} t
$$

Rearrange terms and obtain

$$
\frac{(g(t)-\sqrt{\pi})^{\prime}}{g(t)-\sqrt{\pi}}=-2 t
$$

Solving (3.6) for $g(t)$, we have

$$
g(t)=\int_{0}^{\infty} K(t, s) d s=\frac{\sqrt{\pi}}{2}\left(2-e^{-t^{2}}\right)<\sqrt{\pi}
$$

(2) There exists $\delta=\frac{1}{2}>0$, such that

$$
a(t)-b(t)=\frac{(t+1)^{2}}{t+2}-\frac{t^{2}+t}{2 e(t+2)} \geq \frac{1}{2}=\delta>0, \quad t \in[0, \infty) .
$$

By (i) of Theorem 2.1, we have $|x(t)| \leq \frac{\bar{c}}{\delta}+M=2 e+M, t \geq 0$.

Take $\kappa=\frac{1}{2} \in(0,1)$, it is easy to see that

$$
\kappa a(t)-b(t)=\frac{1}{e} \cdot \frac{(t+1)^{2}}{t+2} \geq \frac{1}{4}>0, \quad t \in[0, \infty) .
$$

By (ii) of Theorem 2.1, for any given $\epsilon>0$, there exists $\widetilde{t}=\widetilde{t}(M, \epsilon)>0$, such that $|x(t)| \leq$ $\frac{\bar{c}}{\delta}+\epsilon=2 e+\epsilon, t \geq \widetilde{t}>0$.

Taking $c(t) \equiv 0$, we have $|x(t)| \leq \epsilon, t \geq \widetilde{t}>0$. So the zero solution of the system (3.1) is stable. 
Example 2 Consider the delay dynamic equation

$$
\left\{\begin{array}{l}
x^{\Delta}(t)=-a(t) x^{\sigma}(t)+b(t) x(t-\tau(t))+c(t), \quad t \in\left[t_{0},+\infty\right)_{\mathbb{T}}, \\
x(t)=|\varphi(t)| \text { for } t \in\left(-\infty, t_{0}\right]_{\mathbb{T}}
\end{array}\right.
$$

where $\varphi(t)$ is bounded, rd-continuous for $t \leq t_{0}$ and $\sup _{t \leq t_{0}}|\varphi(t)|=M, a(t), b(t), c(t), \tau(t)$ are nonnegative, rd-continuous functions for $t \in\left[t_{0}, \infty\right)_{\mathbb{T}}$ and $\sup _{t \geq t_{0}} c(t)=\bar{c}$.

If there exists $\delta>0$ such that

$$
a(t)-b(t) \geq \delta>0 \quad \text { for } t \geq t_{0} .
$$

Similar to Example 1, we get

$$
y^{\Delta}(t) \leq \frac{1}{1+\mu(t) a(t)}\left\{-a(t) y(t)+b(t) \sup _{t-\tau(t) \leq s \leq t} y(s)+c(t)\right\}, \quad t \in\left[t_{0},+\infty\right)_{\mathbb{T}} .
$$

In particular, we take $\mathbb{T}=\mathbb{N}$, (3.7) reduces to

$$
\triangle x(n)=-a(n) x(n+1)+b(n) x(n-2)+c(n), \quad n \geq 2 .
$$

Let $a(n)=2(n+1), b(n)=\frac{n^{2}}{2 n+1}, c(n)=\frac{5 n}{\sqrt[n]{n !}}, \tau(n)=2$.

Obviously, $a(n), b(n)$ are unbounded for $n \in \mathbb{N}$ and $\sup _{n \in \mathbb{N}} c(n)=\lim _{n \rightarrow \infty} \frac{5 n}{\sqrt[n]{n !}}=\bar{c}=5 e$.

(1) $\forall n \geq 2, \frac{a(n)-b(n)}{1+a(t)}=\frac{2(n+1)-\frac{n^{2}}{2 n+1}}{2 n+3}=\frac{3 n^{2}+6 n+2}{(2 n+1)(2 n+3)} \geq \frac{2}{3}=\delta$.

(2) Take $\kappa=0.9 \in(0,1)$, it is easy to see that

$$
\frac{\kappa a(n)-b(n)}{1+a(n)}>0
$$

By (ii) of Theorem 2.1, for any given $\epsilon>0$, there exists $\widetilde{t}=\widetilde{t}(M, \epsilon)>0$, such that $|x(t)| \leq$ $\frac{\bar{c}}{\delta}+\epsilon=\frac{15 e}{2}+\epsilon, t \geq \widetilde{t}>0$.

Taking $c(n) \equiv 0$, we have $|x(t)| \leq \epsilon, t \geq \tilde{t}>0$. So the zero solution of the system (3.7) is stable.

\section{Competing interests}

The authors declare that they have no competing interests.

\section{Authors' contributions}

All authors contributed equally and significantly in writing this article. All authors read and approved the final manuscript.

\section{Author details}

${ }^{1}$ School of Mathematics and Computation Science, Lingnan Normal University, Zhanjiang, 524048, China. ${ }^{2}$ Department of Mathematics, Guangdong University of Petrochemical Technology, Maoming, 525000, China. ${ }^{3}$ School of Mathematics, Sun Yat-Sen University, Guangzhou, 510275, China.

\section{Acknowledgements}

The fourth author was supported by the National Natural Science Foundation of China (No. 11271380) and the Guangdong Province Key Laboratory of Computational Science.

Received: 29 July 2016 Accepted: 22 November 2016 Published online: 01 December 2016

\section{References}

1. Wen, L, Yu, Y, Wang, W: Generalized Halanay inequalities for dissipativity of Volterra functional differential equations. J. Math. Anal. Appl. 347, 169-178 (2008) 
2. Humphries, AR, Stuart, AM: Runge-Kutta methods for dissipative and gradient dynamical systems. SIAM J. Numer. Anal. 31, 1452-1485 (1994)

3. Halanay, A: Differential Equations: Stability, Oscillations, Time Lags. Academic Press, New York (1966)

4. Temam, R: Infinite-Dimensional Dynamical Systems in Mechanics and Physics. Springer Appl. Math. Sci. Ser., vol. 68. Springer, Berlin (1988)

5. Baker, CTH, Tang, A: Generalized Halanay inequalities for Volterra functional differential equations and discretized versions, Invited plenary talk. In: Volterra Centennial Meeting, UTA Arlington, June (1996)

6. Adivar, M, Bohner, EA: Halanay type inequalities on time scales with applications. Nonlinear Anal. 74, 7519-7531 (2011)

7. Bohner, M, Peterson, A: Dynamic Equations on Time Scales: An Introduction with Applications. Birkhäuser, Boston (2001)

8. Bohner, M: Some oscillation criteria for first order delay dynamic equations. Far East J. Appl. Math. 18, 289-304 (2005)

9. Bohner, M, Peterson, AC (eds.): Advances in Dynamic Equations on Time Scales. Birkhäuser, Boston (2003)

Submit your manuscript to a SpringerOpen ${ }^{\odot}$ journal and benefit from:

- Convenient online submission

Rigorous peer review

- Immediate publication on acceptance

- Open access: articles freely available online

- High visibility within the field

- Retaining the copyright to your article 